\title{
Is a handful of genes responsible for the common starling invasion success?
}

\author{
Soraia Barbosa ${ }^{1}$ \\ ${ }^{1}$ University of Idaho
}

October 28,2020

Is a handful of genes responsible for the common starling invasion success?

Invasive species have the ability to colonize new habitats across distinct areas of the globe, rapidly adjusting to new biotic and abiotic conditions, and often experiencing little impact from the decrease in effective population size and genetic diversity. Still, as each invading population represents a subsample of the original native distribution, it is frequent to see variability in terms of the genetic makeup of invading populations and consequently differences in invasion success rates across their non-native range (Blackburn et al. 2017). Invasion success results largely from a combination of the genetic diversity in the native populations, the number of founders and founder events that are effectively get established at the introduction sites, the presence of particularly important genes or new interactions between genes, and genotype $\mathrm{x}$ environment associations (GEAs) (Lee 2002; Blackburn et al. 2017). From the genetic side of the process, invasive populations undergo dramatic changes compared to their native range due to a combination of genetic bottlenecks from the source population with genetic drift at both the introduction site and at the expansion front as the species invades new habitat (Dlugosch \& Parker 2008). Most differences are observed at the neutral level, as quick range expansions lead to an increase in frequency of random neutral variants at the range expansion front, termed allele surfing (Excoffieret al. 2009). But allele surfing might also lead to an increase in frequency of maladapted alleles, which might limit the expansion success of invading populations (Peischl \& Excoffier 2017).

The common starling (Sturnus vulgaris ) is an example of a worldwide invasive species that has been introduced most successfully from its Palearctic range into three other continents including North America, Africa and Australia. Still, the success of the introductions seems to vary between introduction sites. The biggest difference in introduction success might be that found between North America and Australia. Starling invasion of North America started with an introduction of 160 birds in the east coast in 1890s that rapidly expanded across all of North America, establishing populations in the west coast within 50 years (Bodt et al. 2020). In Australia, while starlings were introduced at five locations about sixteen times since the $19^{\text {th }}$ century, only two resulted in established populations (Bodt et al. 2020), suggesting the existence of stronger barriers to expansion than in North America. In a From the Cover article in this issue of Molecular Ecology, Stuart and Cardilini et al. (2020) used Genotyping-by-Sequencing to explore how landscape and environmental heterogeneity shaped the genetic population structure and adaptation of the common starling multiple invasions of Australia, and compare it to the patterns observed in North America, examined in Hofmeister et al. (2019). Stuart and Cardilini et al. (2020) determined that starlings in Australia are distributed across three distinct environmental groups characterized by temperature and precipitation in 'arid', 'semi-arid' and 'non-arid'. However, neutral and adaptive population structure was incongruent with the defined environmental subdivision, rather showing a pattern of isolation-by-distance (IBD). This pattern was further supported by the levels of genetic diversity and divergence across populations. Genetic diversity decreased with distance from the introduction sites in SE Australia, and genetic differentiation between populations increased with geographic distance (Figure 1A and 1B). These patterns contrast with those observed 
at other regions of the world where starlings have been introduced (Hofmeister et al. 2019; Bodt et al. 2020). The starling invasion of North America showed a genetic pattern not consistent with IBD, but rather with isolation-by-environment. In accordance to the rapid continent wide expansion, Hofmeister et al. (2019) also found very low differentiation between sampling localities, indicating not only high dispersal ability but also a panmictic population across the entire North America. Studies on invasion genetics clearly indicate that multiple introductions lead to increased genetic diversity, which in turn provide increased chances to expand into more diverse areas (more genomic targets of selection) (Dlugosch \& Parker 2008; Blackburnet al. 2017). However, despite multiple successful introductions in Australia vs. a single introduction in North America, the extent of the invasion is smaller in Australia and the levels of population differentiation were found to be higher. The authors propose that despite the higher genetic diversity, there could also be greater constraints for gene flow, potentially attributed to environmental (dis)similarities of the different introduction sites in Australia with the native range. The North American climate is more similar to that of the native range of the starling in the Palearctic, and thus it would be expected that population structure would be more evident in Australia where individuals would have to adapt to more extreme conditions than they were adapted in the native range (Colautti \& Lau 2017). To understand the role of environmental variability on expansion, both studies performed outlier-scans and GEAs to identify loci under selection. Contrarily to initial expectations, outlier scans failed at providing strong evidence of selection in association with population structure or environmental variability. In the case of North American starling, this could result from a low genetic diversity across populations, approaching what would be expected in panmixia. In the case in Australian starlings, even in the presence of population structure, outlier detection might simply reflect stochastic patterns as a result of allele surfing. Using redundancy analyses to detect GEAs, both studies found evidence of adaptation to temperature and precipitation. Stuart and Cardilini et al. (2020) found associations of the populations found in 'arid' regions with environmental variables that relate to higher and less variable temperatures, and with increased variability in precipitation (Figure 1C and 1D). Associations with the other environmental regimes ('semi-arid' and 'non-arid') were not as clear, although subdivision of populations in the GEA analysis space appeared to match neutral population structure for these populations. Similarly, Hofmeister et al. (2019) also detected associations between population structure and environmental variables, particularly mean annual temperature.

One of the most interesting results that emerges from the combined analysis of both studies is the identification of similar loci under selection in both Australia and North America. Two genes were reported as candidates for selection in both studies, and both are related to cell structure regulation, suggesting a potential association with cell viability in variable and extreme environments. As noted by many other studies, successful invasions might only need to encompass a few genes that then become important for adaptation to novel conditions (Lee 2002; Nadeau \& Urban 2019). Selection in just a few key loci can be easily spread in species with high mobility, also benefiting from allele surfing, leading to a rapid increase in frequency at the range expansion front and potentially across the entire invasive range. This might explain how such distinct invasion histories as those found across the starling populations around the world led to contradicting expectations in terms of invasiveness (Bodt et al. 2020). Still, the role of other mechanisms such as plasticity, genomic rearrangements, epigenetic variation, among others, still needs to be further examined to fully explain the differences in starling invasion dynamics (Bock et al. 2017). The results provided by Stuart and Cardilini et al.(2020) evidenced that particularly advantageous loci can be positively selected multiple times, across multiple introduction events and through very distinct geographic contexts, despite low effective population sizes and various demographic scenarios. This manuscript suggests that the common starling invasion success around the world has been greatly impacted by a hand full of genes that allow adaptation to extreme environmental conditions. But additional research in other less successful introduction sites should provide further evidence into the mechanisms involved in the invasion success and allow to build a more comprehensive picture of the mechanisms involved.

\section{References}

Blackburn TM, Lockwood JL, Cassey P (2017) The influence of numbers on invasion success. In: Invasion Genetics: the Baker and Stebbins Legacy (eds Barrett S, Colautti R, Dlugosch K, Rieseberg L), pp. 25-39. 
John Wiley \& Sons, Ltd, Chichester, West Sussex, UK.

Bock DG, Caseys C, Cousens RD et al. (2017) What we still don't know about invasion genetics. In: Invasion Genetics: the Baker and Stebbins Legacy (eds Barrett S, Colautti R, Dlugosch K, Rieseberg L), pp. 346-368. Chichester, West Sussex, UK.

Bodt LH, Rollins LA, Zichello JM (2020) Contrasting mitochondrial diversity of European starlings (Sturnus vulgaris ) across three invasive continental distributions. Ecology and Evolution ,10 , 10186-10195.

Colautti R, Lau JA (2017) Contemporary evolution during invasion: evidence for differentiation, natural selection, and local adaptation. In: Invasion Genetics: the Baker and Stebbins Legacy (eds Barrett SCH, Colautti RI, Dlugosch KM, Rieseberg LH), pp. 101-121. John Wiley \& Sons, Ltd, Chichester, West Sussex, UK.

Dlugosch KM, Parker IM (2008) Founding events in species invasions: Genetic variation, adaptive evolution, and the role of multiple introductions. Molecular Ecology , 17 , 431-449.

Excoffier L, Foll M, Petit RJ (2009) Genetic consequences of range expansions. Annual Review of Ecology, Evolution, and Systematics ,40, 481-501.

Hofmeister NR, Werner SJ, Lovette IJ (2019) Environment but not geography explains genetic variation in the invasive and largely panmictic European starling in North America. bioRxiv ,8 , 55 .

Lee CE (2002) Evolutionary genetics of invasive species. Trends in Ecology and Evolution , 17 , 386-391.

Nadeau CP, Urban MC (2019) Eco-evolution on the edge during climate change. Ecography , 42 , 1280-1297.

Peischl S, Excoffier L (2017) Expansion load: recessive mutations and the role of standing genetic variation. In: Invasion Genetics: the Baker and Stebbins Legacy (eds Barrett S, Colautti R, Dlugosch K, Rieseberg L), pp. 213-231. John Wiley \& Sons, Ltd, Chichester, West Sussex, UK.

Stuart KC, Cardilini APA, Cassey P et al. (2020) Signatures of selection in a recent invasion reveal adaptive divergence in a highly vagile invasive species. Molecular Ecology, 29, XXX-XXX.

\section{Hosted file}

Barbosa_etal_News\&Views_Figure.pdf available at https://authorea.com/users/370724/articles/ 489303-is-a-handful-of-genes-responsible-for-the-common-starling-invasion-success 